\title{
Article \\ Sensitivity Improvement of Surface Plasmon Resonance Biosensors with GeS-Metal Layers
}

\author{
Yue Jia ${ }^{1}$, Yunlong Liao ${ }^{2}$ and Houzhi Cai ${ }^{1, *}$
}

1 Key Laboratory of Optoelectronic Devices and Systems of Ministry of Education and Guangdong Province, College of Physics and Optoelectronic Engineering, Shenzhen University, Shenzhen 518060, China; onlyjiayue@hotmail.com

2 International Collaborative Laboratory of 2D Materials for Optoelectronic Science \& Technoloy of Ministry of Education, Shenzhen University, Shenzhen 518060, China; yl.liao@outlook.com

* Correspondence: hzcai@szu.edu.cn

check for updates

Citation: Jia, Y.; Liao, Y.; Cai, H. Sensitivity Improvement of Surface Plasmon Resonance Biosensors with GeS-Metal Layers. Electronics 2022, 11, 332. https://doi.org/10.3390/ electronics11030332

Academic Editor: Nicola Francesco Lopomo

Received: 1 December 2021

Accepted: 19 January 2022

Published: 21 January 2022

Publisher's Note: MDPI stays neutral with regard to jurisdictional claims in published maps and institutional affiliations.

Copyright: (C) 2022 by the authors. Licensee MDPI, Basel, Switzerland. This article is an open access article distributed under the terms and conditions of the Creative Commons Attribution (CC BY) license (https:// creativecommons.org/licenses/by/ $4.0 /)$.

\begin{abstract}
Surface plasmon resonance (SPR) biosensors, with germanium sulfide (GeS) as a sensitive medium and $\mathrm{Al} / \mathrm{Ag} / \mathrm{Au}$ as the metal layers, are reported as we aim to improve the sensitivities of the biosensors. The sensitivities in conventional SPR biosensors, consisting of only metal $\mathrm{Al}, \mathrm{Ag}$, and $\mathrm{Au}$ layers, are $111^{\circ} / \mathrm{RIU}, 117^{\circ} / \mathrm{RIU}, 139^{\circ} / \mathrm{RIU}$, respectively. Additionally, these sensitivities of the SPR biosensors based on the GeS-Al, GeS-Ag, and GeS-Au layers have an obvious improvement, resultant of $320^{\circ} / \mathrm{RIU}, 295^{\circ} / \mathrm{RIU}$, and $260^{\circ} / \mathrm{RIU}$, respectively. We also discuss the changing sensing medium $\mathrm{GeS}$ thickness using layer number to describe the scenario which brought about the diversification on the figure of merit (FOM) and optical absorption (OA) performance of the biosensors. These biosensors show obvious improvement of sensitivity and have strong SPR excitation to analytes; we believe that these kind biosensors could find potential applications in biological detection, chemical examination, and medical diagnosis.
\end{abstract}

Keywords: germanium sulfide; SPR; sensor; high sensitivity

\section{Introduction}

Sensing has been considered one of the most promising applications in plasma [1,2] and photonic [3] technology. Plasma sensing was even recently selected by the scientific community in the United States as one of the top ten emerging technologies (2018). At the surface of positive permittivity materials and negative material, metals usually have the resonance excitation of surface plasmon polaritons (SPSs). This is the surface plasmon resonance (SPR) phenomenon [4], and this kind of phenomenon can be used for real-time monitoring and label-free sensing [5]. Biochemical sensors based on SPR technology have many advantageous properties, such as high operation sensitivities, detection accuracy, and biocompatibilities [6-8]. These kinds of sensors have been studied and used for many biological and chemical applications [9], such as medical diagnosis, enzyme detection, and pharmaceuticals [10]. The effective ways to fabricate sensors are to select large specific surface area combined/adsorption materials such as the popular two-dimensional (2D) materials graphene. After graphene was found, 2D materials fascinated much attention due to their special structural and unique physical/chemical properties [11]. These types of characteristics give 2D materials broad application perspective for different sensor fields $[12,13]$. Fortunately, germanium monosulfide $(\mathrm{GeS})$ which is a phosphorene analogue compound was favorably forecasted and manufactured [11]. Furthermore, GeS exhibits higher air stability and electron mobility than phosphorene [14]. In addition to black phosphorus, layered group IV with a phosphorene-like structure-mono-chalcogenides MX $(\mathrm{M}=\mathrm{Ge}, \mathrm{Sn} ; \mathrm{X}=\mathrm{S}, \mathrm{Se})$ are a class of emerging 2D materials [15-18]. Due to the characteristics of MX series compounds such as excellent mechanical flexibility, high light absorption, high thermoelectric coefficient, high carrier mobility, earth abundance, low toxicity, and 
dynamic stability, they are considered prospective optoelectronic and electronic device materials [19-24]. Li et al. revealed that the high carrier mobility of the GeS monolayer is approximately $3680 \mathrm{~cm}^{2} \mathrm{~V}^{-1}$, which is much higher than that of the $\mathrm{MoS}_{2}$ monolayer [25]. Xue et al. revealed a process for the preparation of GeS film which has a high absorption coefficient $\left(105 \mathrm{~cm}^{-1}\right)$, a suitable band gap $(1.14 \mathrm{eV})$, and $p$ conductivity by in situ selfregulation, which can be used for thin-film photovoltaic devices.

As shown in Figure 1, the single layer GeS geometries, including the top view and side view, are similar to silene and blue phosphorene. Ge atoms and $\mathrm{S}$ atoms are combined and arranged alternately to form a hexagonal buckled. The optimal lattice constant and Ge-S bond length of single-layer GeS is $3.495 \AA$ After structural relaxation, which agrees well with previously reported data.

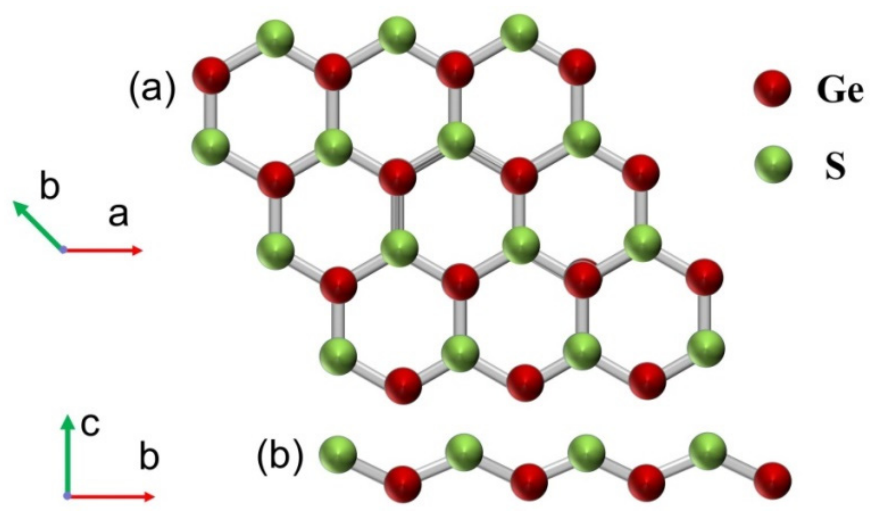

Figure 1. Three-dimensional representation of the structure of GeS: (a) top view (b) side view.

Two dimensional material GeS used as sensing medium in biosensors are reported in this paper. A comparison of three metals, $\mathrm{Al} / \mathrm{Ag} / \mathrm{Au}$, as metal layers are also investigated in these GeS sensors. The sensitivities of the biosensors derived from the GeS-Al, GeS-Ag, and GeS-Au layers are $320^{\circ} / \mathrm{RIU}, 295^{\circ} / \mathrm{RIU}$, and $260^{\circ} / \mathrm{RIU}$, respectively. Additionally, the sensitivities of conventional SPR sensors which do not have $\mathrm{GeS}$ sensing medium, i.e., only based on $\mathrm{Al}, \mathrm{Ag}$, and $\mathrm{Au}$ films, are $111^{\circ} / \mathrm{RIU}, 117^{\circ} / \mathrm{RIU}$, and $139^{\circ} / \mathrm{RIU}$, respectively. The sensitivities have a clear enhancement in these GeS based biosensors. We also discussed the changing of the sensing medium GeS thickness using layer number to describe brought about the diversification on the figure of merit $(F O M)$ and optical absorption $(\mathrm{OA})$ performance of the biosensors. These types of biosensors have doughty SPR excitation, high detection accuracy, and high sensitivity to analytes; they will play a central role in photoelectron applications.

\section{Design Considerations and Theoretical Model}

Z. Lou et al. have synthesized nanoparticles-organic clusters (NOCs) and used them as signal amplification reagents, gaining four times higher SPR signal compared to the sandwich format [26,27]. A highly sensitive sensor with a separate GeS layer is proposed in our design. In our SPR sensors, we put an $\mathrm{Al} / \mathrm{Ag} / \mathrm{Au}$ film on the surface of a $B K 7$ coupling prism, and then put the GeS layer as a biological sensing layer on the top of the $\mathrm{Al} / \mathrm{Ag} / \mathrm{Au}$ layer, seen in Figure 2. BK7 glass is coupling prism, and the GeS monolayer thickness is $0.524 \mathrm{~nm}[28,29]$. As an SPP primary metal, an $\mathrm{Al} / \mathrm{Ag} / \mathrm{Au}$ film of $50 \mathrm{~nm}$ is selected [30]. The $B K 7$ glass refractive index can be expressed [30] using the next equation:

$$
n_{B K 7}=\left(\frac{1.03961212 \lambda^{2}}{\lambda^{2}-0.00600069867}+\frac{0.231792344 \lambda^{2}}{\lambda^{2}-0.0200179144}+\frac{1.03961212 \lambda^{2}}{\lambda^{2}-103.560653}+1\right)^{\frac{1}{2}}
$$




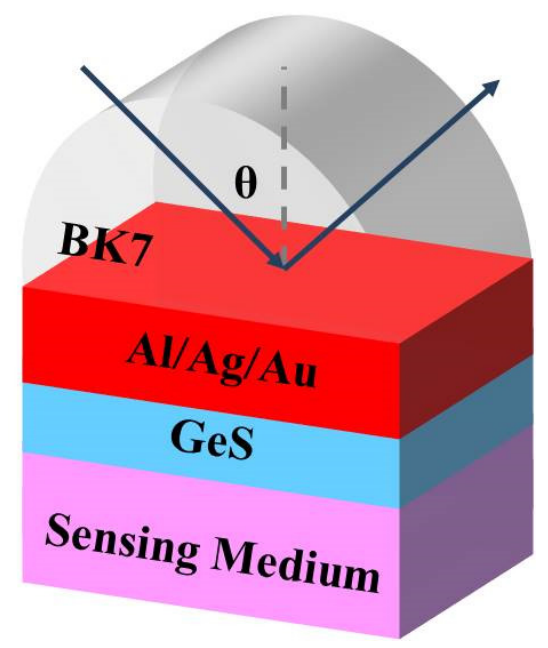

Figure 2. Diagrammatic presentation of the GeS combined on $\mathrm{Al} / \mathrm{Ag} / \mathrm{Au} \mathrm{SPR}$ biochemical sensors for improved sensitivity.

The refractive index of $\mathrm{Ag} / \mathrm{Al} / \mathrm{Au}$ can be calculated using the Drude-Lorentz model [31]:

$$
n_{m}=\sqrt{\varepsilon_{m}}=\left[1-\frac{\lambda^{2} \lambda_{c}}{\lambda_{p}^{2}\left(\lambda_{c}+i \lambda\right)}\right]^{\frac{1}{2}}
$$

Here, $\lambda_{p}$ and $\lambda_{c}$ are the plasma and collision wavelength. For Al layer sensor, the data of $\lambda_{c}$ and $\lambda_{p}$ are $4.0852 \times 10^{-5} \mathrm{~m}$ and $1.3617 \times 10^{-7} \mathrm{~m}$; for Ag they are $1.7614 \times 10^{-5} \mathrm{~m}$ and $1.4541 \times 10^{-7} \mathrm{~m}$; and for Au are $8.9342 \times 10^{-6} \mathrm{~m}$ and $1.6826 \times 10^{-7} \mathrm{~m}$, respectively. Additionally, in our sensor structures, we induced 2D material GeS to put on the surface of $\mathrm{Al}, \mathrm{Ag}$, or $\mathrm{Au}$ to prevent oxidation in order to improve the sensitivities of our sensors.

The GeS refractive index is composed of real and imaginary parts [32], and it is expressed $n_{s}=1.33+\Delta n$ in the wavelength of $633 \mathrm{~nm}$. The expression represents the refractive index of the target medium, where $\Delta n$ indicates the refractive index variation caused by biological action or chemical reaction.

The reflectivity is studied by the transfer matrix method. The TM polarized light $N$-layer model which using matrix method is effective and accurate. The SPR sensor simulation is calculated using Matrix\&Laboratory (MATLAB) software. In our GeS based biosensors, $\mathrm{GeS}$ and $\mathrm{Al} / \mathrm{Ag} / \mathrm{Au}$ layers are stacked in the $B K 7$ glass-coupling prism vertically, and each layer is named for the dielectric constant $\left(\varepsilon_{k}\right)$, refractive index $\left(n_{k}\right)$, and thickness $\left(d_{k}\right)$. The resonance angle is the incident angle corresponding to the minimum reflectivity. The tangential field at the first boundary $Z=Z_{1}=0$ is related to the tangential field at the final boundary $Z=Z_{N-1}$ :

$$
\left[\begin{array}{l}
U_{1} \\
V_{1}
\end{array}\right]=M\left[\begin{array}{l}
U_{N-1} \\
V_{N-1}
\end{array}\right]
$$

where $V$ and $U$ indicate the magnetic fields and electric fields components of the boundary surface, respectively. $M$ represents the feature matrix of the composite architecture and $k$ represents the $k$ th layer in the $N$-layer model. $p$-polarized light corresponds to:

$$
\begin{gathered}
\prod_{K=2}^{N-1} M_{K}=\left[\begin{array}{ll}
M_{11} & M_{12} \\
M_{21} & M_{22}
\end{array}\right] \\
M_{K}=\left[\begin{array}{cc}
\cos \beta_{K} & \frac{-\sin \beta_{K}}{q_{k}} \\
-i q_{k} \sin \beta_{k} & \cos \beta_{k}
\end{array}\right]
\end{gathered}
$$




$$
\begin{gathered}
q_{k}=\left(\frac{\mu_{k}}{\delta_{k}}\right)^{\frac{1}{2}} \cos \theta_{k}=\frac{\left(\varepsilon_{k}-n_{1}^{2} \sin \theta_{1}^{2}\right)^{\frac{1}{2}}}{\varepsilon_{k}} \\
\beta_{k}=\frac{2 \pi}{\lambda} n_{k} \cos \theta_{k}\left(Z_{k}-Z_{k-1}\right)=\frac{2 \pi d_{k}}{\lambda}\left(\varepsilon_{k}-n_{1}^{2} \sin \theta_{1}^{2}\right)^{\frac{1}{2}}
\end{gathered}
$$

Through mathematical simplifications, reflection coefficient $r_{p}$ of the $p$-polarized light $N$-layer complex was obtained, and the corresponding amplitude reflection coefficient $\left(R_{p}\right)$ can be achieved by the square of $r_{p}$ :

$$
\begin{gathered}
r_{p}=\frac{\left(M_{11}+M_{12} q_{5}\right) q_{1}-\left(M_{21}+M_{22} q_{5}\right)}{\left(M_{11}+M_{12} q_{5}\right) q_{1}+\left(M_{21}+M_{22} q_{5}\right)} \\
R_{p}=\left|r_{p}\right|^{2}
\end{gathered}
$$

The sensitivities, expressed as SR1 $=\Delta \theta / \Delta n$, cause the refractive index of the sensing medium $(\Delta n)$ to change, leading to the shift in the resonance angle $(\Delta \theta)$ [33]. The detection accuracy $(D A)$ is the ratio of the resonance angle shift in $\left(\Delta \theta_{\text {res }}\right)$ in the reflectance curve to the full width at half-maximum value (FWHM):

$$
D A=\Delta \theta / F W H M
$$

The FOM is the specific value of the sensitivity $(S)$ to the $F W H M$ in the reflectance curve [34]:

$$
F O M=S / F W H M
$$

\section{Results and Discussion}

We selected BK7 glass as the coupling prism for our designed GeS biochemical sensors, due to its low refractive index [35]. Traditional SPR sensors, such as the Kretschmann geometry biochemical sensors, mainly include a simple metal layer to measure the SPPs, as shown in Figure 3. The sensitivities of conventional Al, Ag, and Au metals are $111^{\circ} / \mathrm{RIU}$, $117^{\circ} / \mathrm{RIU}$, and $139^{\circ} / \mathrm{RIU}$, respectively. When using the $B K 7$ glass prism, they are shown in Figure $3 a, c, e$, respectively. However, these values are not ideal for the sensitivity of biochemical sensors. To improve the sensitivity of the sensor, we developed an SPR biochemical sensor using GeS material. We put the addition of the GeS material on the surface of metallic $\mathrm{Al} / \mathrm{Ag} / \mathrm{Au}$ layer, and then the detection target flow past the 2D material surface in the structure, using this way to increase the sensitivity of the biosensors. In Figure $3 b, d, f$, the charts show that the sensitivities are greatly improved when the GeS material is introduced in the previous sensor structure. The sensitivities of these sensors obtained for the $\mathrm{Al}, \mathrm{Ag}$, and $\mathrm{Au}$ metal layers are $320^{\circ} / \mathrm{RIU}, 295^{\circ} / \mathrm{RIU}$, and $260^{\circ} / \mathrm{RIU}$ when the GeS layers accumulating to 33,28 , and 18 layers, respectively.

In the following experiments, $2 \mathrm{D}$ GeS material can use the liquid phase stripping (LPE) method to prepare and then use the spin coating method to coat on the $\mathrm{Al} / \mathrm{Ag} / \mathrm{Au}$ layers $(\sim 50 \mathrm{~nm})$ of the $B K 7$ coupling prism. Using a refractive index matching solution, the $\mathrm{GeS}-\mathrm{Al} / \mathrm{Ag} / \mathrm{Au}$ multilayer film can be connected with to $B K 7$ prism to construct the SPR sensor.

Table 1 shows the changes in the number of GeS layers $(L)$, maximum sensitivity $(S)$, $F O M, F W H M$, resonance angle change $(\Delta \theta)$, and $D A$ data for the GeS based on $\mathrm{Al} / \mathrm{Ag} / \mathrm{Au}$ sensors. The thickness of $\mathrm{Al}, \mathrm{Ag}$, and $\mathrm{Au}$ was set to $50 \mathrm{~nm}$. The incident angles were set from $0^{\circ}$ to $90^{\circ}$. The thickness of the GeS layer was obtained from reference [36], and the number of layers was calculated based on the single-layer thickness of GeS, which was $0.375 \mathrm{~nm}$. With the increasing number of GeS layers, the reflectivity curve presents a larger peak sensitivity angle. The maximum sensitivity is achieved when the optimal numbers of $\mathrm{GeS}$ layers are added. For $\mathrm{GeS}$ on $\mathrm{Al}, \mathrm{Ag}$, and $\mathrm{Au}$, the sensitivity reaches its maximum when the number of layers is 33,28 , and 18 , respectively. Due to the limitation of a certain angle range, the change of resonance angle is directly related to the sensitivity; 
for example, the sensitivity decreases after the optimized GeS layer number is exceeded with the decrease in the resonance angle. Finally, we also realized that the SPR line width increases and that FOM decrease will improve the sensor sensitivities [37]. The refractive index of $B K 7$ at $\lambda=633 \mathrm{~nm}$ is calculated as 1.515 . The refractive index of the sensing medium can change, increasing if the prism reflection angle has a lower refractive index; this indicates that higher sensitivity will be apparent with a lower prism refractive index. Therefore, a $B K 7$ prism is selected as the coupling prism in our GeS sensor structures to improve the sensitivities.

(a) $\mathrm{Al}$

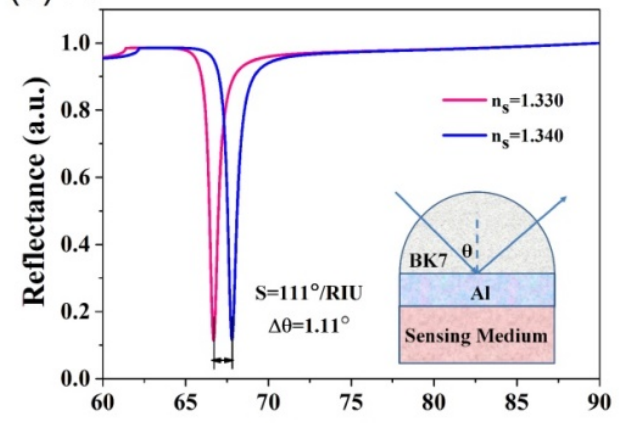

(c) $\mathrm{Ag}$

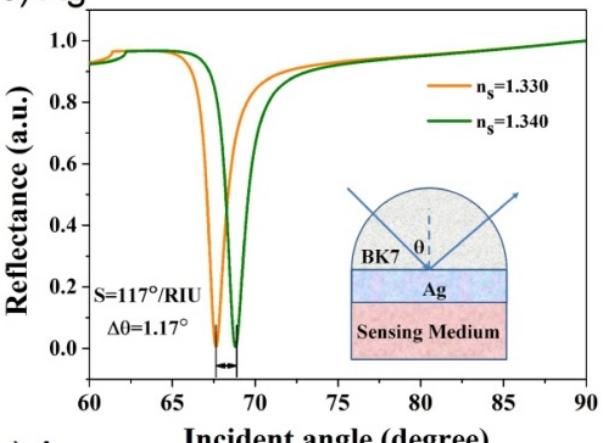

(e) $\mathrm{Au}$

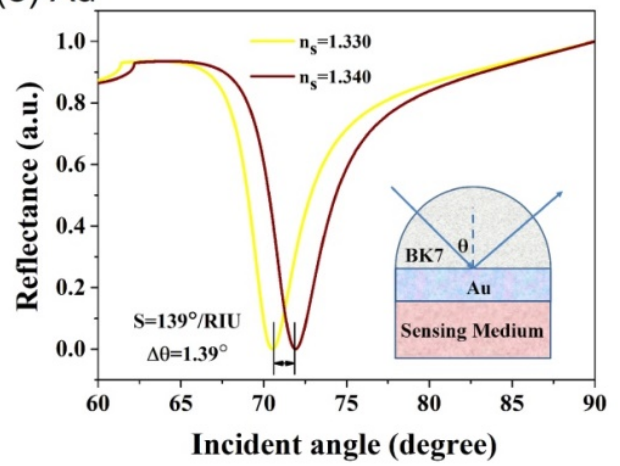

(b) Al

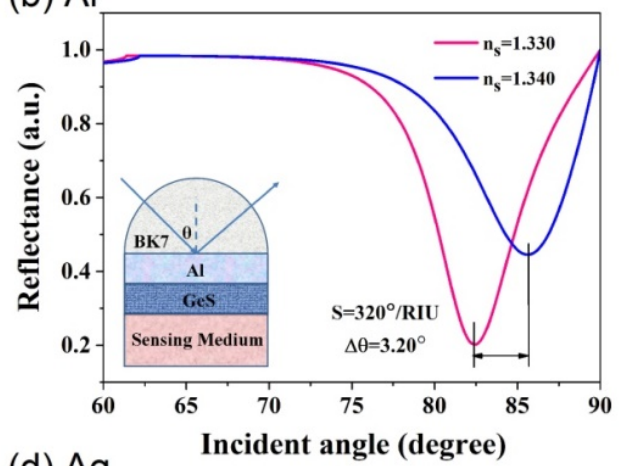

(d) $\mathrm{Ag}$

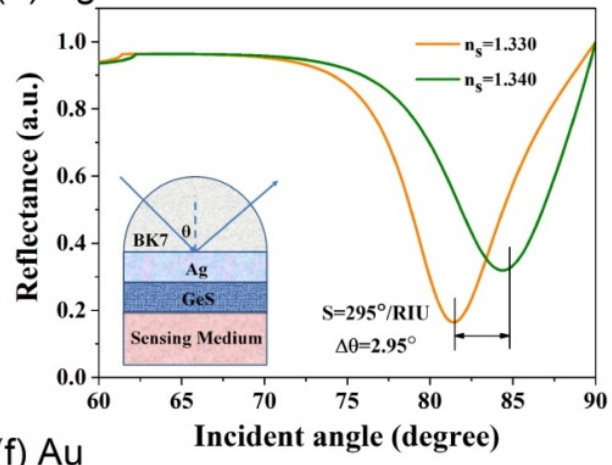

(f) $\mathrm{Au}$

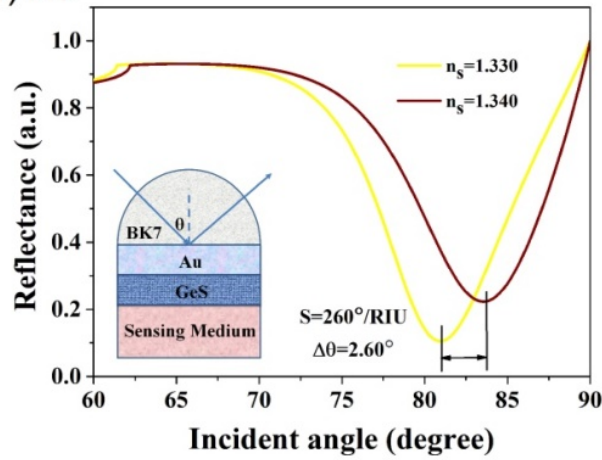

Figure 3. (a,c,e) The incident angle reflectance change of the conventional sensors with single $\mathrm{Al} / \mathrm{Ag} / \mathrm{Au}$ metal layer, and $(\mathbf{b}, \mathbf{d}, \mathbf{f})$ The incident angle reflectance change of the sensors with GeS on top of $\mathrm{Al} / \mathrm{Ag} / \mathrm{Au}$ metal layer.

The properties of the sensors are also impacted by the GeS layer numbers. Figure 4 shows the changes in $D A, F O M$, and FWHM, and sensitivity with the number of GeS layers at $633 \mathrm{~nm}$, with the incident angle ranging from $34.65^{\circ}$ to $34.85^{\circ}$. DA is a key factor to reflect the functions of biosensors. As can be seen from the curves in Figure $4 a$, as the number of GeS layers changes between 1 and 35,DA presents a downward trend. This is due to the application of a germanium sulfide layer to widen the reflection curve. Although $D A$ decreases with the increase in GeS layers, $D A$ remains high. In Figure $4 b, F O M$ is the specific value of the sensitivity to the FWHM in the reflectance curve. GeS-Au based biosensor FOM augments as the number of GeS layers increase, and the GeS-Al and GeS-Ag based 
biosensors do not exhibit significant change. Figure 4c shows that GeS layer numbers have an important effect on reflectivity. With increasing GeS layer numbers, the inclination of the SPR reflectivity curve becomes shallower and the FWHM widens due to the absorption of the GeS layers. Figure $4 \mathrm{~d}$ indicates the change in the peak sensitivities of the SPR biosensors based on GeS-Al, GeS-Ag, and GeS-Au at the wavelength of $633 \mathrm{~nm}$ with the number of GeS layers (L). The SPR biosensors based on GeS-Au have higher sensitivity than GeS-Al and GeS-Ag SPR biosensors when they are at the same layers. The SPR response of $\mathrm{Al}$ and Ag layers is more sensitive than that of Au layers, but the chemical stability is lower. The GeS layer is an important part of the SPR sensor, which can prevent the oxidation of the oxidizable $\mathrm{Al} / \mathrm{Ag} / \mathrm{Au}$ metal, enhance the absorption of the sensing medium, and improve the stability and biocompatibility of the biosensor structure.

Table 1. Performances of the Proposed GeS-Based Sensors.

\begin{tabular}{|c|c|c|c|c|c|c|}
\hline $\begin{array}{c}\text { Type of } \\
\text { Metal Layer }\end{array}$ & $\begin{array}{c}\text { Optimized } \\
\text { Layer Number }(L)\end{array}$ & $\begin{array}{c}\text { Sensitivity }(S) \\
\left({ }^{\circ} / \mathrm{RIU}\right)\end{array}$ & $F O M$ & $F W H M$ & $\Delta \theta$ & $D A$ \\
\hline \multirow{3}{*}{$\mathrm{Al}$} & 0 & 111 & 54.4 & 0.49 & 1.11 & 2.040 \\
\hline & 15 & 141 & 219.9 & 1.56 & 1.41 & 0.641 \\
\hline & 33 & 320 & 1088.4 & 3.39 & 3.20 & 0.294 \\
\hline \multirow{3}{*}{$\mathrm{Ag}$} & 0 & 117 & 89.3 & 1.31 & 1.17 & 0.763 \\
\hline & 15 & 158 & 75.5 & 1.68 & 1.58 & 0.595 \\
\hline & 28 & 295 & 66.2 & 4.45 & 2.95 & 0.224 \\
\hline \multirow{3}{*}{$\mathrm{Au}$} & 0 & 139 & 36.6 & 3.79 & 1.39 & 0.263 \\
\hline & 10 & 233 & 35.3 & 4.02 & 1.84 & 0.248 \\
\hline & 18 & 260 & 33.4 & 6.97 & 2.60 & 0.143 \\
\hline
\end{tabular}

(a)

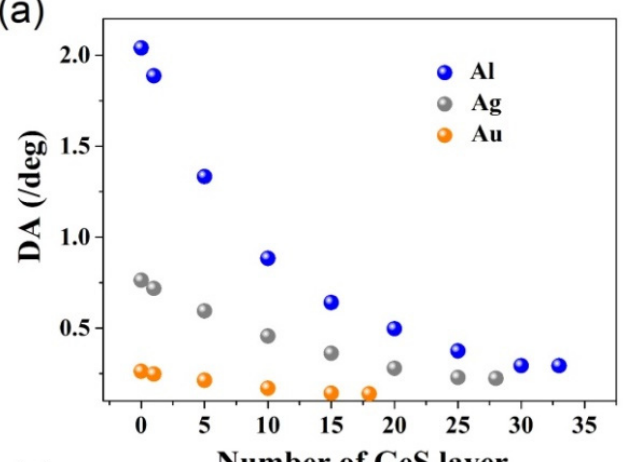

(c)

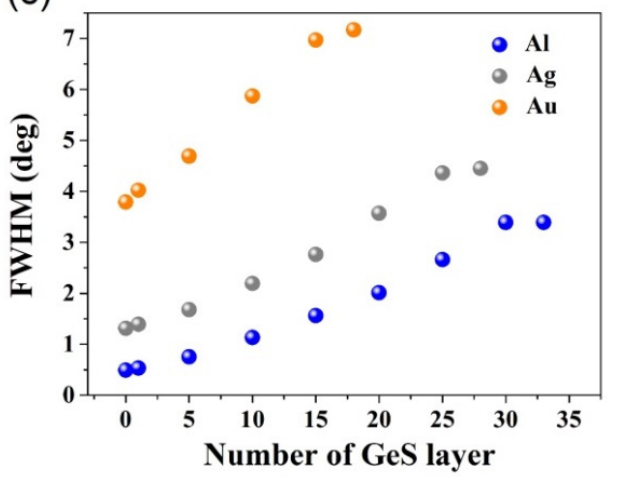

(b)
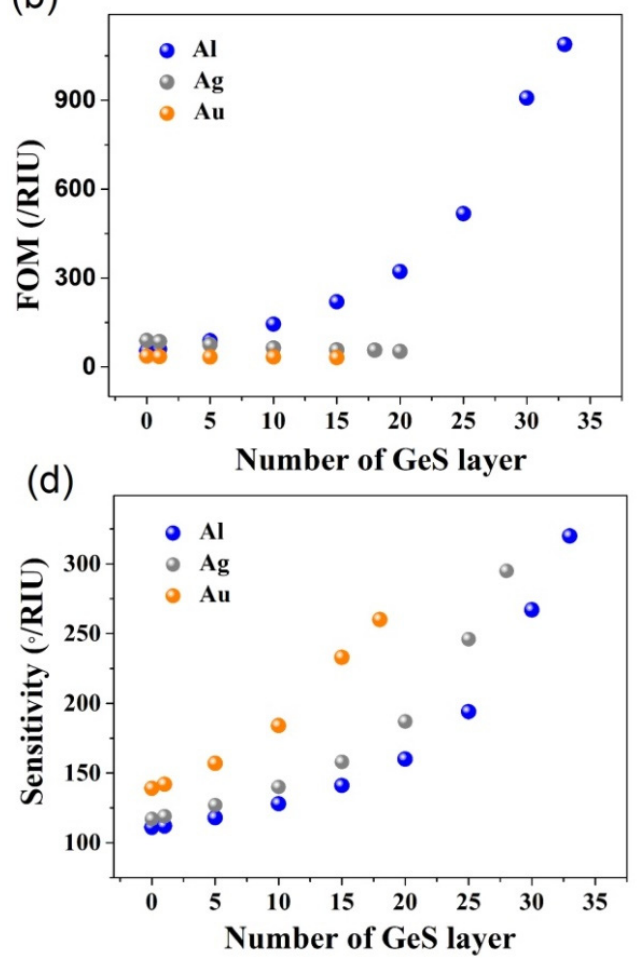

Figure 4. Variation in (a) DA, (b) FOM, (c) FWHM, and (d) sensitivities changing with numbers of GeS layers for the proposed SPR biosensors. 
The electric field distributions corresponding to the resonance angles are shown in Figure 5a,c,e. When the detection target refractive index is from 1.33 to 1.36 , the electric field at the GeS/sending medium interface obviously varies dramatically. This indicates that the GeS based sensors are sensitive to small changes when checking detection target. When the refractive index of the detection medium on the contact surface changes a little bit, the surface wave property will have a large change, and then it will lead to a change in the electric field. Figure $5 b, d, f$ shows that the changes in reflectance with the incident angles increase with increasing detection target refractive index, and the incident angles corresponding to the resonance angles increase. From $n_{s}=1.33$ to $n_{s}=1.36$, the resonance angles are $75.205^{\circ}, 77.145^{\circ}, 79.395^{\circ}$, and $82.150^{\circ}$, respectively.
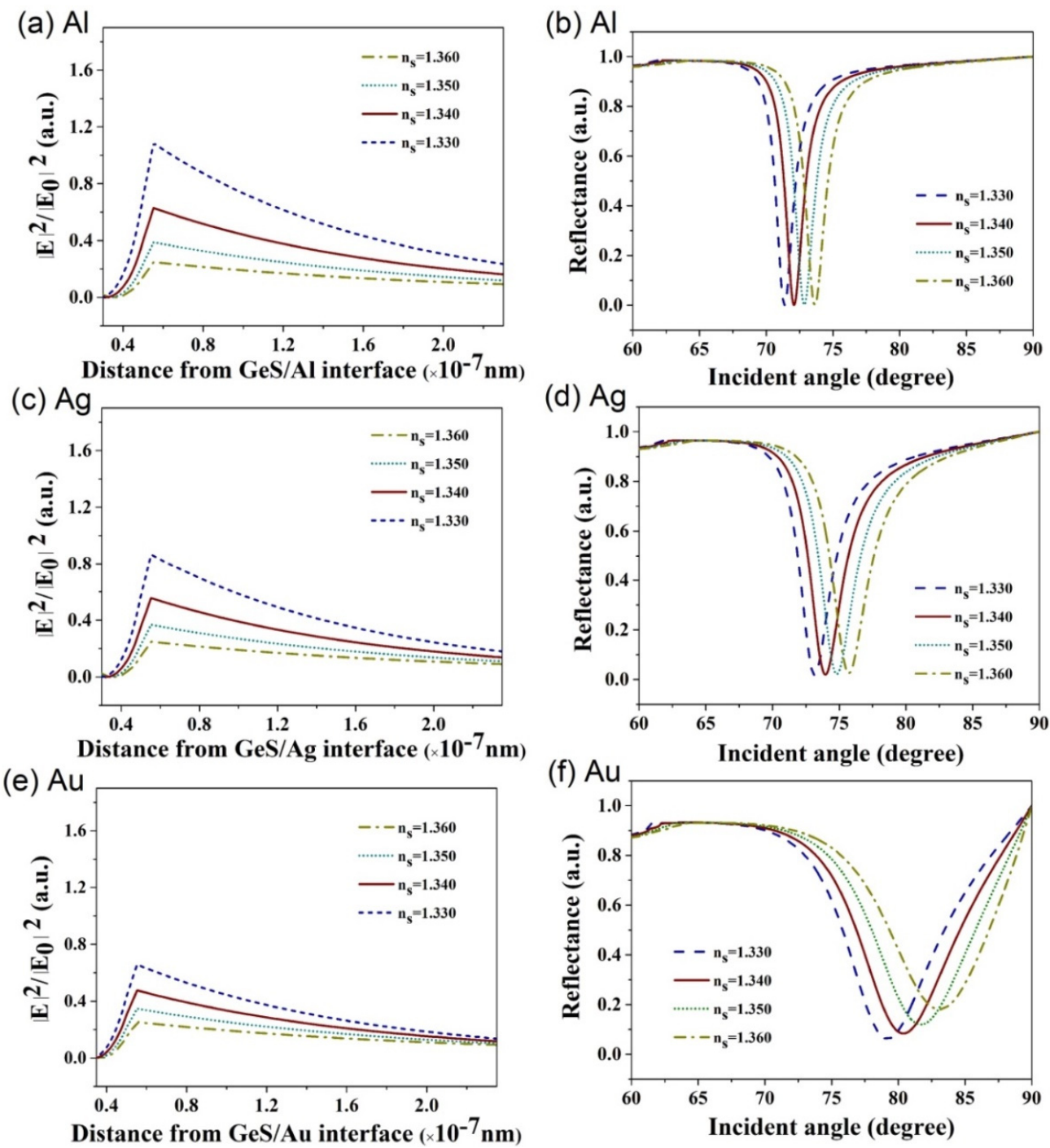

Figure 5. (a,c,e) The electric field distribution of the GeS based sensor and $(\mathbf{b}, \mathbf{d}, \mathbf{f})$ variation in reflectance with the incident angle of the sensor based on few-layer GeS for $n_{S}=1.33$ to $n_{s}=1.36$.

\section{Conclusions}

In this study, different kinds of GeS based sensors, designed to enhance sensitivity, are reported. Three kinds of metal, $\mathrm{Al} / \mathrm{Ag} / \mathrm{Au}$, were used as metal layers for comparison in these GeS sensors. Compared with the conventional biosensor, which only has metal layers, the sensitivities of proposed GeS based biosensors show a large improvement. The highest sensitivities for our sensors, based on GeS-Al, GeS-Ag, and GeS-Au films, are $320^{\circ} / \mathrm{RIU}, 295^{\circ} / \mathrm{RIU}$, and $260^{\circ} / \mathrm{RIU}$, respectively; conventional SPR sensors, only based on $\mathrm{Al} / \mathrm{Ag} / \mathrm{Au}$ films, exhibited sensitivities of $111^{\circ} / \mathrm{RIU}, 117^{\circ} / \mathrm{RIU}$, and $139^{\circ} / \mathrm{RIU}$, 
respectively. The two dimensional material GeS layer not only prevented the oxidizable metals from oxidizing, enhancing the surface plasmons of metal, but also enhanced the absorption of biomolecules. The changing of the sensing medium GeS thickness, using numbers of layers, brought about the diversification on the figure of merit (FOM) and optical absorption (OA) performance of the biosensors. From these simulation results, the present sensors display high sensitivities and strong SPR excitations analytes. With these outstanding characteristics, these GeS based structure sensors will have a remarkable effect in the field of electrical optical sensing technology.

Author Contributions: Conceptualization and Writing Original Draft, Y.J.; Investigation, Y.L.; Project administration, H.C. All authors have read and agreed to the published version of the manuscript.

Funding: This work is supported by National Natural Science Foundation of China (NSFC) (11775147); Guangdong Basic and Applied Basic Research Foundation (Nos. 2019A1515011474 and 2019A1515110130); Shenzhen Science and Technology Program (Nos. JCYJ20210324095007020, JCYJ20200109105201936 and JCYJ20190808115605501).

Conflicts of Interest: The authors declare no conflict of interest.

\section{References}

1. Mayer, K.M.; Hafner, J.H. Localized Surface Plasmon Resonance Sensors. Chem. Rev. 2011, 111, 3828-3857. [CrossRef] [PubMed]

2. Špačková, B.; Wrobel, P.; Bocková, M.; Homola, J. Optical Biosensors Based on Plasmonic Nanostructures: A Review. Proc. IEEE 2016, 104, 2380-2408. [CrossRef]

3. Xu, D.; Xiong, X.; Wu, L.; Ren, X.-F.; Png, C.E.; Guo, G.-C.; Gong, Q.; Xiao, Y.-F. Quantum plasmonics: New opportunity in fundamental and applied photonics. Adv. Opt. Photon. 2018, 10, 703-756. [CrossRef]

4. Ouyang, Q.; Zeng, S.; Jiang, L.; Qu, J.; Dinh, X.-Q.; Qian, J.; He, S.; Coquet, P.; Yong, K.-T. Two-Dimensional Transition Metal Dichalcogenide Enhanced Phase-Sensitive Plasmonic Biosensors: Theoretical Insight. J. Phys. Chem. C 2017, 121, $6282-6289$. [CrossRef]

5. Srivastava, S.K.; Verma, R.; Gupta, B.D. Theoretical modeling of a self-referenced dual mode SPR sensor utilizing indium tin oxide film. Opt. Commun. 2016, 369, 131-137. [CrossRef]

6. Sajad, P.; Fardin, M.N. Simultaneous analysis of some volatile compounds in food samples by array gas sensors based on polypyrrole nano-composites. Sens. Rev. 2017, 37, 155-164.

7. Mohammad, A.S.-M.; Sajad, P. Nanostructured Conducting Polymer/Copper Oxide as a Modifier for Fabrication of L-DOPA and Uric Acid Electrochemical Sensor. Electroanalysis 2016, 28, 2075-2080.

8. Anooshe, G.-D.; Mohammad, T.A.; Ashkan, G.-D. Quick, Single-Frequency Dielectric Characterization of Blood Samples of Pediatric Cancer Patients by a Cylindrical Capacitor: Pilot Study. Electronics 2020, 9, 1-16.

9. Liedberg, B.; Nylander, C.; Lunström, I. Surface plasmon resonance for gas detection and biosensing. Sens. Actuators 1983, 4, 299-304. [CrossRef]

10. Zeng, S.; Baillargeat, D.; Ho, H.-P.; Yong, K.-T. Nanomaterials enhanced surface plasmon resonance for biological and chemical sensing applications. Chem. Soc. Rev. 2014, 43, 3426-3452. [CrossRef]

11. Zhang, S.; Yan, Z.; Shengli, Z.; Chen, Z.; Zeng, H. Atomically Thin Arsenene and Antimonene: Semimetal-Semiconductor and Indirect-Direct Band-Gap Transitions. Angew. Chem. Int. Ed. 2015, 54, 3112-3115. [CrossRef] [PubMed]

12. Wang, S.-G.; Tan, C.-J.; Yang, Q.; Xu, Y.-X.; Li, S.-L.; Chen, X.-P. A Novel Ultra-Sensitive Nitrogen Dioxide Sensor Based on Germanium Monosulfide Monolayer. IEEE Electron. Device Lett. 2017, 38, 1590-1593. [CrossRef]

13. Cui, S.; Pu, H.; Wells, S.A.; Wen, Z.; Mao, S.; Chang, J.; Hersam, M.C.; Chen, J. Ultrahigh sensitivity and layer-dependent sensing performance of phosphorene-based gas sensors. Nat. Commun. 2015, 6, 8632. [CrossRef] [PubMed]

14. Zhang, S.; Zhou, W.; Ma, Y.; Ji, J.; Cai, B.; Yang, S.; Zhu, Z.; Chen, Z.; Zeng, H. Antimonene Oxides: Emerging Tunable Direct Bandgap Semiconductor and Novel Topological Insulator. Nano Lett. 2017, 17, 3434-3440. [CrossRef] [PubMed]

15. Zhu, Z.; Tománek, D. Semiconducting Layered Blue Phosphorus: A Computational Study. Phys. Rev. Lett. 2014, 112, 176802. [CrossRef]

16. Zhu, Z.; Guan, J.; Liu, D.; Tománek, D. Designing Isoelectronic Counterparts to Layered Group V Semiconductors. ACS Nano 2015, 9, 8284-8290. [CrossRef]

17. Liu, H.; Neal, A.T.; Zhu, Z.; Luo, Z.; Xu, X.; Tomanek, D.; Ye, P.D. Phosphorene: An Unexplored 2D Semiconductor with a High Hole Mobility. ACS Nano 2014, 8, 4033-4041. [CrossRef]

18. Zhao, H.; Mao, Y.; Mao, X.; Shi, X.; Xu, C.; Wang, C.; Zhang, S.; Zhou, D. Band Structure and Photoelectric Characterization of GeSe Monolayers. Adv. Funct. Mater. 2018, 28, 1704855. [CrossRef]

19. Gu, D.; Tao, X.; Chen, H.; Zhu, W.; Ouyang, Y.; Peng, Q. Enhanced photocatalytic activity for water splitting of blue-phase GeS and GeSe monolayers via biaxial straining. Nanoscale 2019, 11, 2335-2342. [CrossRef] 
20. Yap, W.C.; Yang, Z.; Mehboudi, M.; Yan, J.-A.; Barraza-Lopez, S.; Zhu, W. Layered material GeSe and vertical GeSe/MoS 2 p-n heterojunctions. Nano Res. 2018, 11, 420-430. [CrossRef]

21. Rikta, K.A.; Anower, M.S.; Rahman, M.S.; Rahman, M.M. SPR biosensor using SnSe-phosphorene heterostructure. Sens. Bio-Sens. Res. 2021, 33, 100442. [CrossRef]

22. Devi, T.N.; Chiu, N.-F. A Review of Graphene-Based Surface Plasmon Resonance and Surface-Enhanced Raman Scattering Biosensors: Current Status and Future Prospects. Nanomaterials 2021, 11, 216.

23. Singh, A.K.; Hennig, R.G. Computational prediction of two-dimensional group-IV mono-chalcogenides. Appl. Phys. Lett. 2014, 105, 042103. [CrossRef]

24. Zhao, P.; Yang, H.; Li, J.; Jin, H.; Wei, W.; Yu, L.; Huang, B.; Dai, Y. Design of new photovoltaic systems based on two-dimensional group-IV monochalcogenides for high performance solar cells. J. Mater. Chem. A 2017, 5, 24145-24152. [CrossRef]

25. Li, F.; Liu, X.; Wang, Y.; Li, Y. Germanium monosulfide monolayer: A novel two-dimensional semiconductor with a high carrier mobility. J. Mater. Chem. C 2016, 4, 2155-2159. [CrossRef]

26. Yuan, C.; Lou, Z.; Wang, W.; Yang, L.; Li, Y. Synthesis of Fe3C@C from Pyrolysis of Fe3O4-Lignin Clusters and Its Application for Quick and Sensitive Detection of PrPSc through a Sandwich SPR Detection Assay. Int. J. Mol. Sci. 2019, 20, 741. [CrossRef]

27. Lou, Z.; Han, H.; Zhou, M.; Wan, J.; Sun, Q.; Zhou, X.; Gu, N. Fabrication of Magnetic Conjugation Clusters via Intermolecular Assembling for Ultrasensitive Surface Plasmon Resonance (SPR) Detection in a Wide Range of Concentrations. Anal. Chem. 2017, 89, 13472-13479. [CrossRef]

28. Hess, P. Thickness of elemental and binary single atomic monolayers. Nanoscale Horiz. 2020, 5, 385-399. [CrossRef]

29. Fan, X.; Su, L.; Zhang, F.; Huang, D.; Sang, D.K.; Chen, Y.; Li, Y.; Liu, F.; Li, J.; Zhang, H.; et al. Layer-Dependent Properties of Ultrathin GeS Nanosheets and Application in UV-Vis Photodetectors. ACS Appl. Mater. Interfaces 2019, 11, 47197-47206. [CrossRef]

30. Dai, X.; Liang, Y.; Zhao, Y.; Gan, S.; Jia, Y.; Xiang, Y. Sensitivity Enhancement of a Surface Plasmon Resonance with Tin Selenide (SnSe) Allotropes. Sensors 2019, 19, 173. [CrossRef]

31. Gupta, B.D.; Sharma, A.K. Sensitivity evaluation of a multi-layered surface plasmon resonance-based fiber optic sensor: A theoretical study. Sens. Actuators B Chem. 2005, 107, 40-46. [CrossRef]

32. Xie, J.; Zhang, D.; Yan, X.-Q.; Ren, M.; Zhao, X.; Liu, F.; Sun, R.; Li, X.; Li, Z.; Chen, S.; et al. Optical properties of chemical vapor deposition-grown $\mathrm{PtSe}_{2}$ characterized by spectroscopic ellipsometry. 2D Mater. 2019, 6, 035011. [CrossRef]

33. Maharana, P.K.; Jha, R. Chalcogenide prism and graphene multilayer based surface plasmon resonance affinity biosensor for high performance. Sens. Actuators B Chem. 2012, 169, 161-166. [CrossRef]

34. Homola, J. Surface Plasmon Resonance Sensors for Detection of Chemical and Biological Species. Chem. Rev. 2008, 108, 462-493. [CrossRef]

35. Wu, L.; Jia, Y.; Jiang, L.; Guo, J.; Dai, X.; Xiang, Y.; Fan, D. Sensitivity Improved SPR Biosensor Based on the MoS $2 /$ GrapheneAluminum Hybrid Structure. J. Lightwave Technol. 2017, 35, 82-87. [CrossRef]

36. Wiedemeier, H.; Georg, H.; Schnering, G.V. Refinement of the structures of GeS, GeSe, SnS and SnSe. Z. Für Krist.-Cryst. Mater. 1978, 148, 295-304. [CrossRef]

37. Jia, Y.; Li, Z.; Wang, H.; Saeed, M.; Cai, H. Sensitivity Enhancement of a Surface Plasmon Resonance Sensor with Platinum Diselenide. Sensors 2020, 20, 131. [CrossRef] 